\title{
The effect study of thunderstorm electric field on the lateral distribution of positrons and electrons at YBJ
}

\author{
Kegu Axi, Xunxiu Zhou*, Bing Zhao, Daihui Huang \\ School of Physical Science and Technology, Southwest Jiaotong University, Chengdu 610031, China \\ E-mail: zhouxx@swjtu.edu.cn
}

\begin{abstract}
For a high altitude experiment, the lateral distribution (particle density distribution) of secondary particles at detector level is an important parameter to get the information of primary shower event. During thunderstorms, the charged particles in extensive air showers will be accelerated or decelerated in strong atmospheric electric field. The intensity and the position of ground cosmic rays could be influenced. In this work, Monte Carlo simulations are performed by using CORSIKA to study the effect of thunderstorms electric field on the lateral distribution of charged particles at YBJ (4300 m a.s.1., Tibet, China). We found that the lateral distribution will be changed significantly in thunderstorm field. And the variation amplitude is found to be highly dependent on the electric field, the zenith angle of the primary shower event and the distance to the shower core. Our simulation results could be helpful in understanding the phenomena observed in ground based experiments (such as ARGO-YBJ) and the physical mechanism of secondary charged particles accelerated by thunderstorm electric field.
\end{abstract}

Keywords: Thunderstorm electric field, Cosmic rays, Lateral distribution, Monte Carlo simulation

36th International Cosmic Ray Conference -ICRC2019-

July 24th - August 1st, 2019

Madison, WI, U.S.A.

\footnotetext{
* Speaker.
} 


\section{Introduction}

When a primary cosmic ray enters the atmosphere, numerous secondary particles, such as positrons, electrons, photons and muons, are created through the process of hadron and electromagnetic cascades. These secondary particles are distributed over a large area around the shower core. The lateral distribution, which is the density distribution of ground cosmic rays around the shower axis at detector level, is crucial to study the information of primary shower event, such as primary components, energy spectrum and the acceleration mechanism. Therefore, scientists have been paying close attention to the lateral distribution of secondary particles.

The Nishimura-Kamata-Greisen (NKG) function [1, 2] gives a good theoretical approach for the lateral distribution of electromagnetic particles, from which the dependency of the lateral growth of cascade on the energy, intensity and type of the primary cosmic rays can be clearly shown. Several ground-based observation experiments, such as ARGO-YBJ [3], KASKADE [4], NBU [5] and Utah neutrino detector [6], have reported the lateral distribution of secondary particles associated to the energy of primary cosmic rays. A few simulations have been done to study the lateral distribution. Apel et al. [4] found that the lateral distributions of electrons and muons was related to the mass, energy and type of the primary shower event. And the same laws were obtained by Zhao [7], Wang [8] and Atreidis [9].

In addition, the influence of the environment is not to be neglected. Sampath et al. [10] indicted that the geomagnetic field and atmospheric air density have considerable effects on the lateral distribution of extensive air shower. During thunderstorms, the magnitude of atmospheric electric field is commonly up to $1000 \mathrm{~V} / \mathrm{cm}$ [11-13]. In such strong fields, the location, time, energy and flux of ground cosmic rays will be changed significantly. It means that the lateral distribution of secondary particles, which produced by primary cosmic rays interact with the atmosphere, will also be inevitably influenced. In this work, Monte Carlo simulations are performed by using CORSIKA to study the effect of near-earth thunderstorm electric field on the lateral distribution of positrons and electrons at YBJ. This paper is organized as follows: The simulation parameters are introduced in Section 2. Simulation results and discussions are shown in Section 3. The conclusions are presented in Section 4.

\section{Simulation paramaters}

The CORSIKA (COsmic Ray SImulations for KAscade) is a Monte Carlo simulation program to study the evolution and properties of EAS in the atmosphere initiated by nuclei, hadrons, photons, or any other particles $[14,15]$. In this work, we use the 7.5700 version of CORSIKA. The selected hadronic interaction model is QGSJET-II-04 (Quark Gluon String model with JETs) for high energy and GHEISHA (Gamma Hadron Electron Interaction SHower code) in the low energy range. The energy cutoff was set to $0.1 \mathrm{MeV}$, below which value positrons and electrons are discarded from the simulation. 
In this work, the electric fields are regarded as homogeneous and the thickness of the field layer is $1000 \mathrm{~m}$, from the detector level $(4300 \mathrm{~m})$ up to $5300 \mathrm{~m}$, corresponding to the atmospheric depth from $606 \mathrm{~g} / \mathrm{cm}^{2}$ to $531 \mathrm{~g} / \mathrm{cm}^{2}$. We assume proton primary with a zenith angle in the interval from $0^{\circ}$ to $60^{\circ}$. Number of showers is $2 \times 10^{4}$. According to the observed energy range of the ARGOYBJ detector, we simulated primary protons with energy from $100 \mathrm{GeV}$ to $1 \mathrm{PeV}$ and power law spectrum of -2.7. The electric fields are chosen as a series of values in the range of $-1000 \mathrm{~V} / \mathrm{cm}$ to $1000 \mathrm{~V} / \mathrm{cm}$. Here, we define the positive electric field as one that accelerates positrons downward in the direction of the earth.

\section{Simulation results and discussions}

In this paper, the effects of thunderstorm electric fields on the lateral distributions of positrons and electrons at YBJ are simulated.

\subsection{The lateral distributions of positrons and electrons in absence of electric field}

Fig. 1 shows the lateral distributions of positions and electrons in absence of electric field at YBJ. We can see that the percent of particle density decreases as the $R$ (the radius of secondary particles to shower core) increases. The average radius can be calculated by

$$
\bar{R}=\sum_{i=1}^{N} R_{i} / N
$$

here $R_{i}$ is the distance of the particle $i$ to shower core at detector level, $N$ is the total number of particles in the shower. In the following figures, the distributions are normalized to the density at $R=2000 \mathrm{~m}$. From Fig.1, we can obtain the average radius $\bar{R}$ of positrons and electrons are 321.1 $\mathrm{m}$ and $340.9 \mathrm{~m}$, respectively. That means the lateral distribution of electrons is wider than that of positrons.

Fig. 2 shows the density distribution of particles as a function of the distance to shower core with different primary zenith angles in absence of electric field. We can see the lateral widens with the higher zenith angle. The average radius are $282.1 \mathrm{~m}\left(\theta \sim 0^{\circ}-20^{\circ}\right), 323.7 \mathrm{~m}\left(\theta \sim 20^{\circ}-40^{\circ}\right)$ and $457.5 \mathrm{~m}\left(\theta \sim 40^{\circ}-60^{\circ}\right)$, respectively.

\subsection{The variations of lateral distributions in thunderstorm fields}

In the field strength of $1000 \mathrm{~V} / \mathrm{cm}$, the distributions of particle density as a function of the distance to shower core are shown in Fig. 3. The result in absence field is plotted just for comparison with the plots in fields. It is easy to see that the lateral distributions become wider in fields. In 1000 $\mathrm{V} / \mathrm{cm}$, the average lateral distribution is $348.1 \mathrm{~m}$, and the increase amplitude is about $4.4 \%$. In $-1000 \mathrm{~V} / \mathrm{cm}$, the average radius is $364.5 \mathrm{~m}$, and the ampitude enhancement is up to $9.3 \%$.

For different primary zenith angles, the distribution of particle density in $1000 \mathrm{~V} / \mathrm{cm}$ and $1000 \mathrm{~V} / \mathrm{cm}$ are shown in Fig. 4 and Fig. 5, respectively. As shown in the two figures, the effects of 


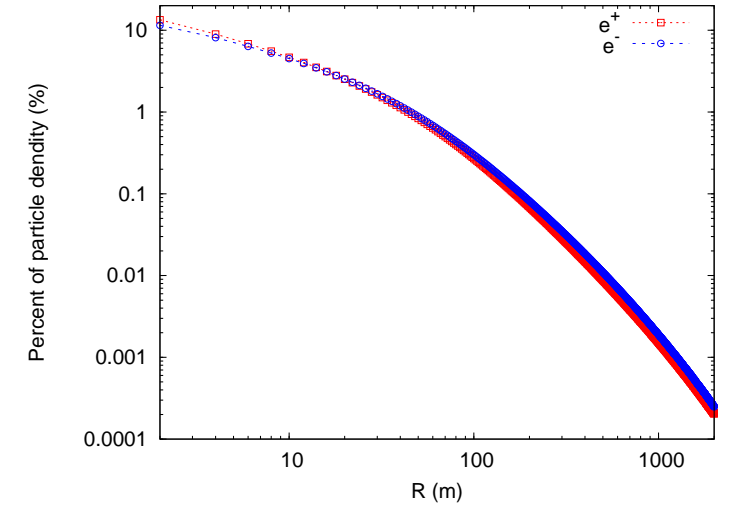

Fig. 1: Lateral distributions as a function of distance to shower core.

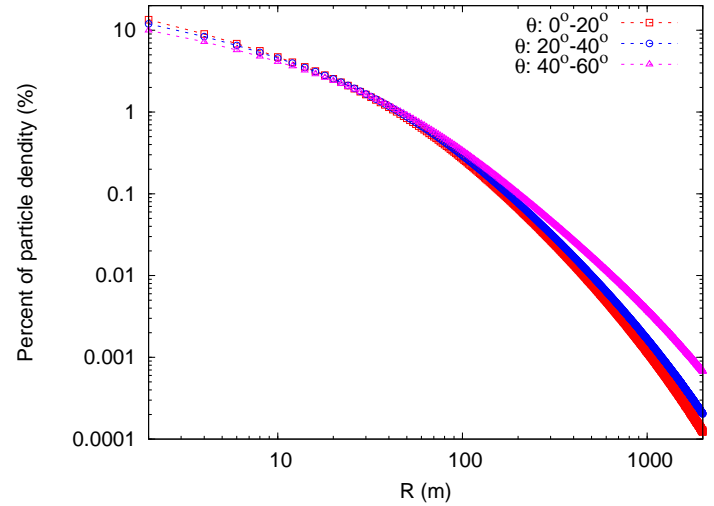

Fig. 2: Lateral distributions as a function of distance to shower core with different primary zenith angles.

electric field on the lateral distribution with different primary zenith angles are different. And the effect becomes larger as the primary zenith angle increases. The average radius in different fields with different primary zenith angles are listed in table 1.

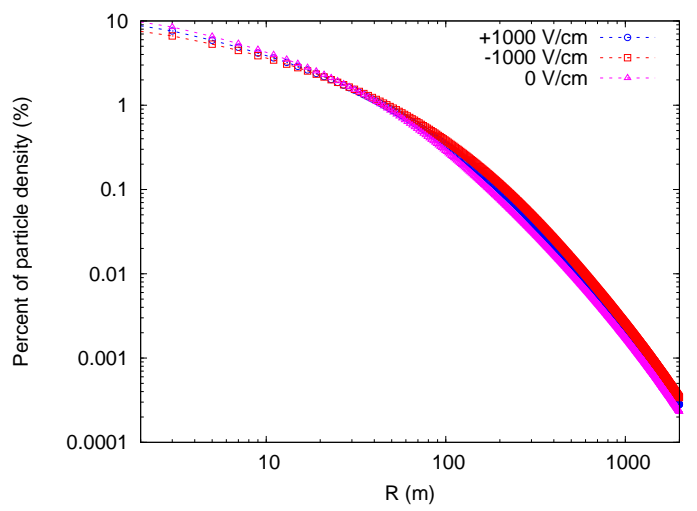

Fig. 3: Lateral distributions as a function of distance to shower core in $\pm 1000 \mathrm{~V} / \mathrm{cm}$.

Table 1: the $\bar{R}$ in different fields with different primary zenith angles

\begin{tabular}{|c|c|c|c|}
\hline $\mathrm{E}(\mathrm{V} / \mathrm{cm})$ & $\theta: 0^{\circ}-20^{\circ}$ & $\theta: 20^{\circ}-40^{\circ}$ & $\theta: 40^{\circ}-60^{\circ}$ \\
\hline 0 & 282.1 & 323.7 & 457.5 \\
\hline+1000 & 286.9 & 337.1 & 489.0 \\
\hline-1000 & 301.0 & 353.6 & 502.1 \\
\hline
\end{tabular}

Fig. 6 shows the average lateral distributions in different electric fields at YBJ and Fig. 7 shows the variations of lateral distributions. We can see that the average radius increases as the electric field strength increasess. The average radius is larger in negative field than that of in the same strength positive field. 


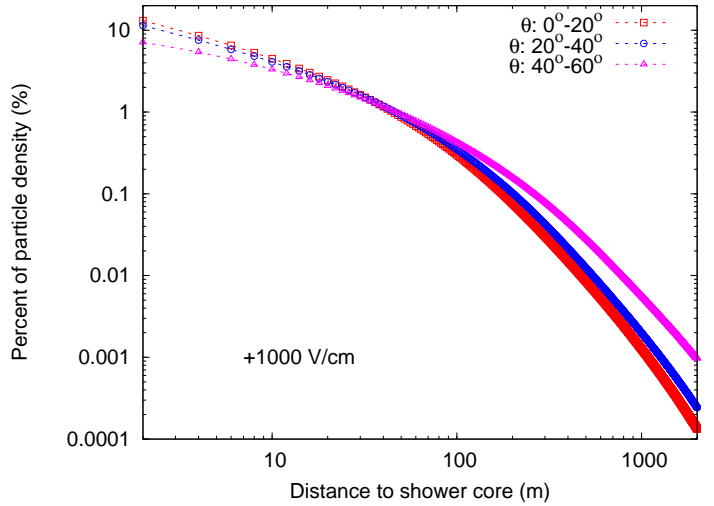

Fig. 4: Lateral distributions as a function of distance to shower core with different primary zenith angles in $+1000 \mathrm{~V} / \mathrm{cm}$.

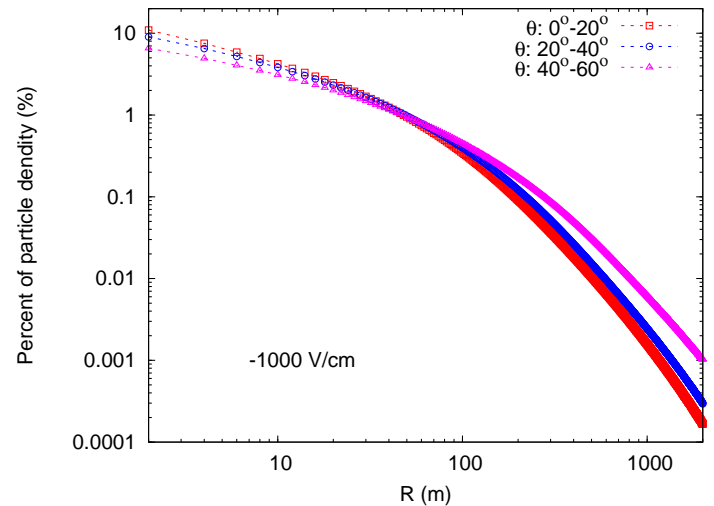

Fig. 5: Lateral distributions as a function of distance to shower core with different primary zenith angles in $-1000 \mathrm{~V} / \mathrm{cm}$.

Fig. 8 and Fig. 9 show the average lateral distributions as a function of electric field with different primary zeninth angles. The average radius graduately increases with increasing field strength. In addition, for the larger zenith angle, the average radius become much larger. That means the enhancement amplitude is higher for showers with larger zenith angles.

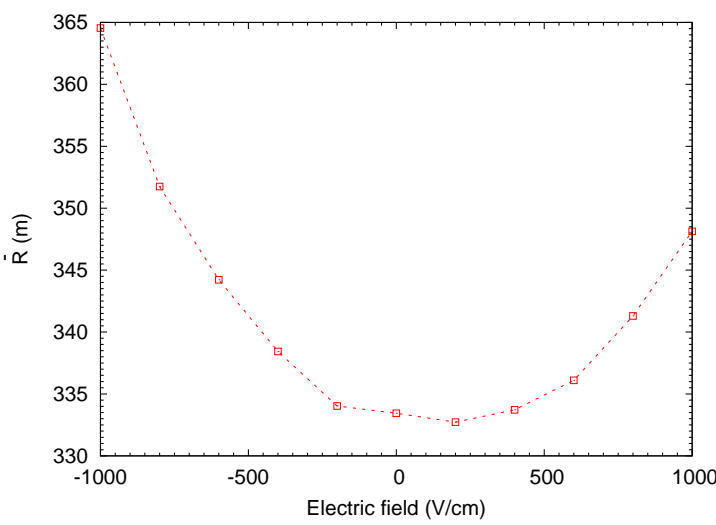

Fig. 6: The distributions of average radius as a function of field.

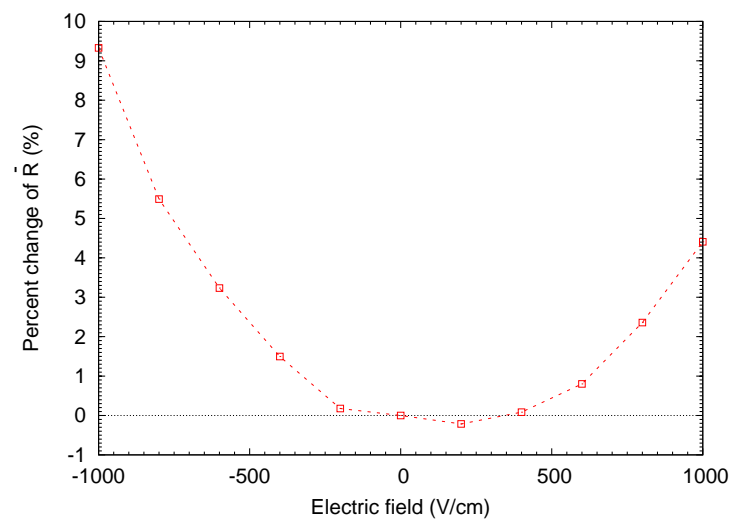

Fig. 7: Percent change of the average radius as a function of field.

\subsection{The variations of counting rate in electric field}

Fig. 10 shows the variations of particle number as a function of the distance to shower core in $\pm 1000 \mathrm{~V} / \mathrm{cm}$. We can see the counting rate decrease in negative fields when $R$ is less than $\sim 10.6 \mathrm{~m}$, otherwise the number increase as the distance to shower core increases. There is a same law in positive field, and the particle number decrease if $R$ is less than $\sim 50.5 \mathrm{~m}$. The amplitude enhancement in a negative field is much larger than that of in positive field with the same strength, and the decrease amplitude is larger in positive field. 


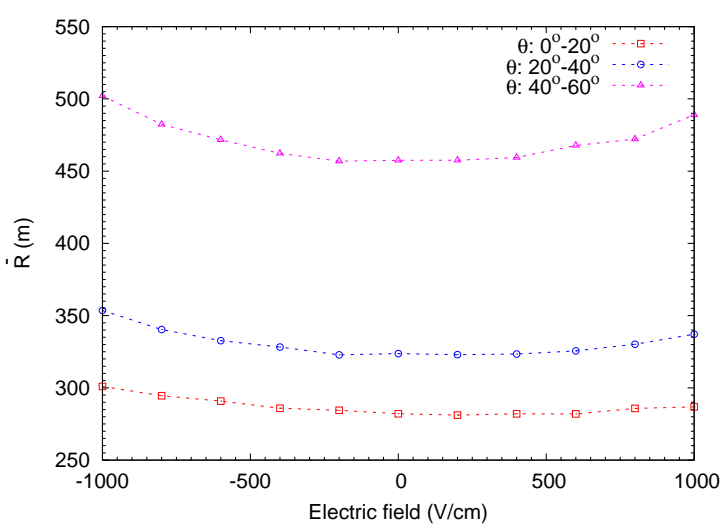

Fig. 8: The distributions of average radius as a function of field with different primary zenith angles.

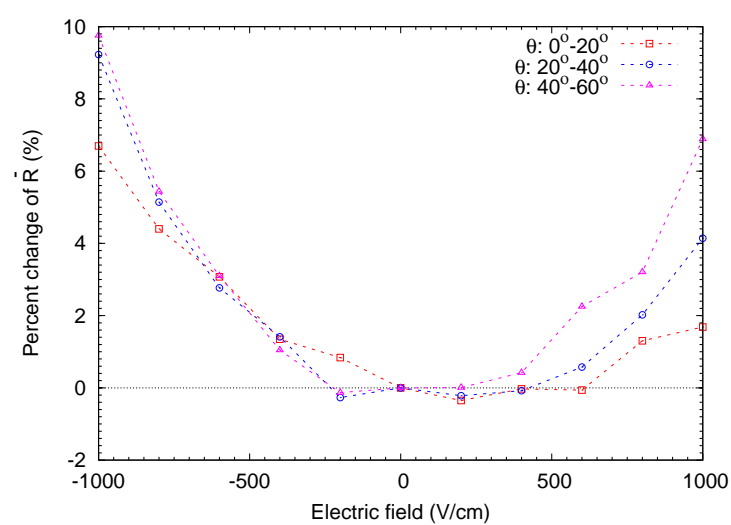

Fig. 9: Percent change of the average radius as a function of field with different primary zenith angles.

Fig. 11 and Fig. 12 show the percent change of particle number for different primary zenith angles in $1000 \mathrm{~V} / \mathrm{cm}$ and $-1000 \mathrm{~V} / \mathrm{cm}$, respectively. We can see the increasing phenomenon appeared in the area far from the shower core, and the amplitude enhancement becomes larger with the higher zenith angle. Howerever, the decreasing phenomenon occurred in lower lateral radius range with higher zenith angle, and the declining amplitude increases with the increasing zenith angle.

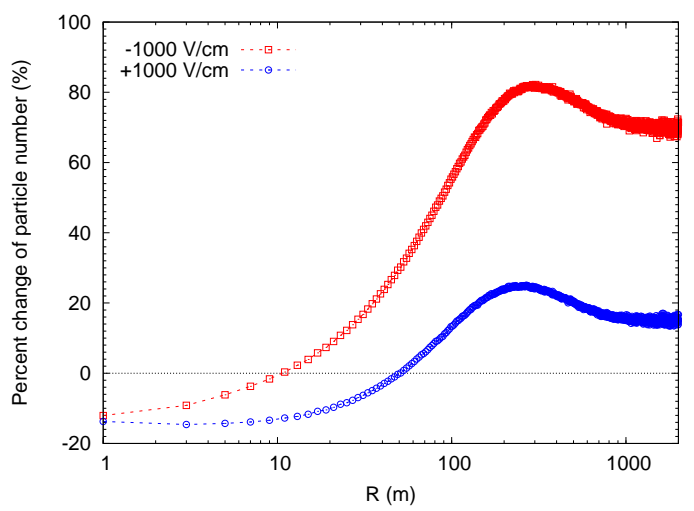

Fig. 10: Percent change of particle number as a function of distance to shower core in $\pm 1000 \mathrm{~V} / \mathrm{cm}$.

From our simulations, we can see the variations of particles number are highly dependent on the detector size. Fig. 13 describes the variations of particle number with different primary zenith angles in different fields at $R \leqslant 60 \mathrm{~m}$. The counting rate increases in negative fields and decreases in positive ones, and the amplitudes depend on the zenith angle. The fluctuations in our results are due to the limited shower number in the Monte Carlo simulation.

At $R \leqslant 2000 \mathrm{~m}$, the number variations as a function of electric field with different primary zenith angles are shown in Fig. 14. In negative electric fields and in positive fields greater than $600 \mathrm{~V} / \mathrm{cm}$, the number increases with increasing field strength, and the amplitude increases with 


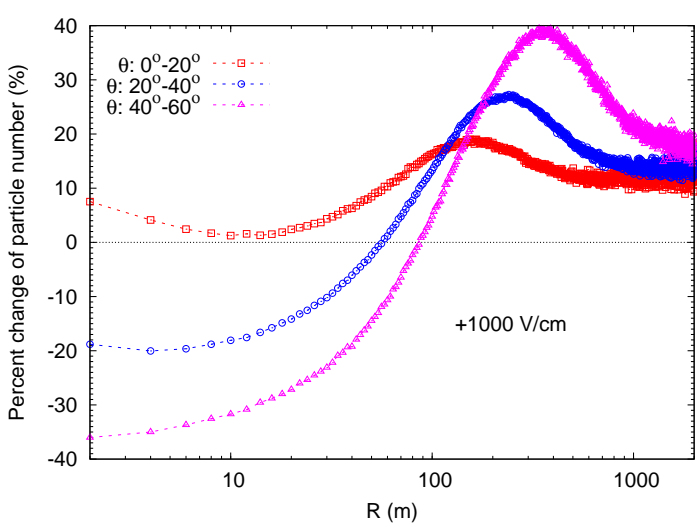

Fig. 11: Percent change of particle number as a function of distance to shower core with different primary zenith angles in $+1000 \mathrm{~V} / \mathrm{cm}$.

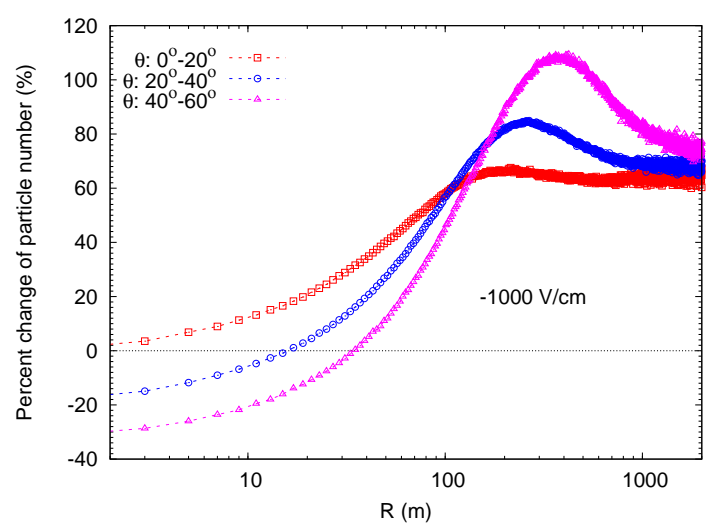

Fig. 12: Percent change of particle number as a function of distance to shower core with different primary zenith angles in $-1000 \mathrm{~V} / \mathrm{cm}$.

the higher zenith angle. The amplitude of the enahncement in negative fields is significantly higher than that in positive ones with the same strength. The decreasing phenomenon occurs in the field range of $0-600 \mathrm{~V} / \mathrm{cm}$ and the maximum amplitude is about $1.52 \%$.

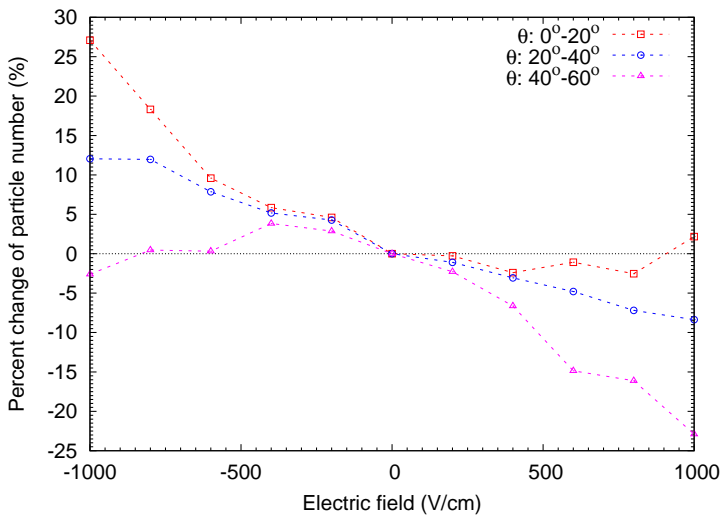

Fig. 13: Percent change of particle number as a function of electric field with different primary zenith angles at $R \leqslant 60 \mathrm{~m}$.

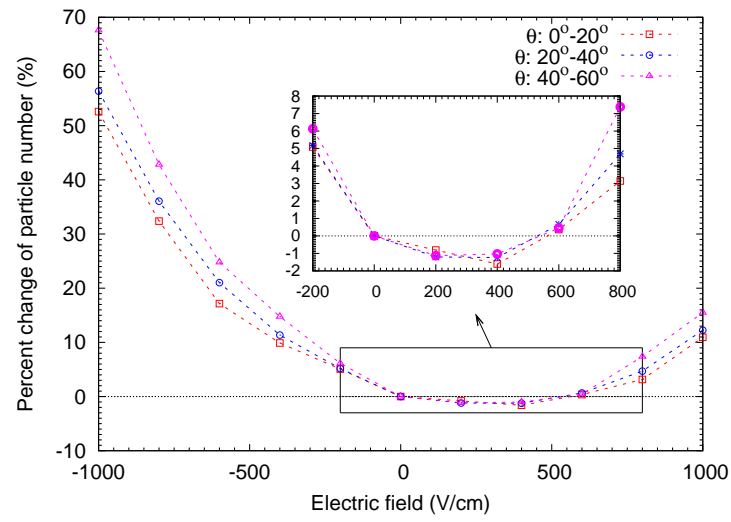

Fig. 14: Percent change of particle number as a function of electric field with different primary zenith angles at $R \leqslant 2000 \mathrm{~m}$.

\section{Conclusions}

In this paper, Monte Carlo simulations are performed with CORSIKA 7.5700 packages to study the effect of near-earth thunderstorm electric field on the lateral distribution of positrons and electrons at YBJ. The simulation results show the lateral distribution widens in field, and the amplitude increases with the increasing strength of the field and the primary zenith angle.

The counting rate variations are highly dependent on the polarity of electric field, the zenith angle of the primary shower event, and the detector size (the distance to shower core). In negative 
field, if the $R$ becomes large enough, the counting rate increases and the amplitude enhancement is larger for higher zenith angle; otherwise, the amplitude enhancement is larger for lower zenith angle. In positive field, if $R$ is less than a certain value, the decreasing phenomenon occurs, and the amplitude becomes larger for higher zenith angle.

The simulation results of field effects on the lateral distributions will be useful in understanding the phenomena observed in ground based experiments.

\section{Acknowledgments}

This work is supported by the National Natural Science Foundation of China (NSFC) under the grant No. 11475141 and 11847307.

\section{References}

[1] K. Kamata et al., The Lateral and the Angular Structure Functions of Electron Showers, Progress of Theoretical Physics Supplement, 6 (1958)

[2] J.G. Wilson et al., Progress in Cosmic Ray Physics, Zeitschrift Naturforschung Teil A, 7 (1952)

[3] B. Bartoli et al., EAS age determination from the study of the lateral distribution of charged particles near the shower axis with the ARGO-YBJ experiment, Astroparticle Physics, 93 (2017)

[4] W.D. Apel et al., Comparison of measured and simulated lateral distributions for electrons and muons with KASCADE, Astroparticle Physics, 24 (2006)

[5] D.K. Basak, N. Chaudhuri, Measurements of the characteristics of low-energy extensive air showers (EAS), Nuovo Cimento C, 9 (1986)

[6] R.B. Coats et al., Lateral distribution of muon pairs in underground cosmic ray showers, Journal of Physics A, 3 , $6(1970)$

[7] J. Zhao et al., EAS lateral distribution measured by analog readout and digital readout of ARGO-YBJ experiment, in proceedings of 34th ICRC, The Hague, The Netherlands, 30 July- 6 August (2015)

[8] H. Wang et al., Lateral distribution of shower particles in high energy family events of cosmic rays [J], High Energy Physics and Nuclear Physics, 27, 3 (2003)

[9] G. Atreidis et al., Numerical study of the electron lateral distribution in atmospheric showers of high energy cosmic rays, American Journal of Modern Physics, 2, 6 (2013)

[10] S. Sampath et al., Development of cosmic ray simulation program: earth cosmic ray shower (ECRS). Georgia State University, (2007)

[11] T.C. Marshall et al., Electrical structure and updraft speeds in thunderstorms over the southern Great Plains[J], Journal of Geophysical Research Atmospheres, 100, D1 (1995)

[12] T.C. Marshall et al., Electric field magnitudes and lightning initiation in thunderstorms, Journal of Geophysical Research Atmospheres, 100, D4 (1995)

[13] T.C. Marshall et al., Observed electric fields associated with lightning initiation [J], Geophysical Research Letters, 32, 3 (2005)

[14] J.N. Capdevielee et al., Extensive air shower simulations with the CORSIKA program[C], in Aip Conference, American Institute of Physics, Jan 1, 276, 545 (1992)

[15] D. Heck et al., CORSIKA: a Monte Carlo code to simulate extensive air showers, Report FZKA: 6019 (1998) 